\title{
Technique for Measuring Spatial Distribution of the Surface Acoustic Wave Velocity in Metals
}

\author{
Oleh MOKRYY, Oleksandr TSYRULNYK
}

Karpenko Physico-Mechanical Institute of the NAS of Ukraine

Naukova 5, 79060 Lviv, Ukraine; e-mail: mokomo@lviv.farlep.net

(received November 18, 2015; accepted August 18, 2016)

\begin{abstract}
In this paper a possibility of determining a local velocity of the surface acoustic Rayleigh waves using a transducer, with the rigidly connected emitting and receiving parts, is considered. A problem on spatial resolution of such a transducer for investigation of inhomogeneous specimens is also examined. A high spatial resolution can be obtained due to the transducer displacement by a value less than the distance between the emitting and receiving parts. It is shown that in this case it is not necessary to measure the transducer displacement with a high accuracy for precise determination of the velocity. Such an effect is obtained through measuring the velocity of surface waves in one local region of the specimen with respect to the other. The criterion for optimal spatial resolution selection during spatially inhomogeneous specimens study is also proposed. The proposed criterion use is illustrated on the example of the determination of spatial distribution of the surface acoustic velocity in a steel specimen subjected to inhomogeneous plastic deformation.
\end{abstract}

Keywords: ultrasound; surface acoustic waves; acoustic wave velocity; spatial resolution.

\section{Introduction}

Properties of acoustic waves in metals depend on different factors: phase composition, structure, mechanical stresses etc. (Hung-YANG, Jung-Ho, 2003; LEWANDOWSKI, 2001). By measuring acoustic properties like velocity, attenuation and scattering of acoustic waves it is possible to get the information about the metal state and to carry out non-destructive testing of different products. It should be noted that very often the velocity of acoustic waves differs in different areas of the studied materials. Spatial inhomogeneity of acoustic properties in metals is caused by plastic deformation, hydrogenation, mechanical stresses, welding and various technological processes. The spatial distribution of acoustic waves velocity allows us to obtain important information about these phenomena in metals (LI, 2013; Murav'Ev et al., 1996). Such measurements require the development of new techniques with high spatial resolution. The spatial resolution is minimum size of area where one can carry out velocity measurement. The measuring value of velocity is averaged in this area.

One of the effective techniques in this respect is the determination of the velocity of the surface acoustic waves (SAW) of the Rayleigh type (Johnson, 1993; MURAV'EV et al.1996; ZHI et al., 2000). Using these waves it is easy to localize the measuring area which is defined by the location of the emitting and receiving transducers. The spatial resolution for these waves is size of measure area in direction parallel to propagation wave. The possibility to localize the measuring areas along the specimen thickness should also be noted, since the depth of SAW penetration in this case is specified by the wave length. Another advantage of the SAW velocity use is that only one specimen surface, where the SAW propagates, is necessary for its measuring. Such investigations can be carried out both in the laboratory and in the manufacturing conditions for the on-line control of different object states. It is worth noting that the changes in the SAW velocity under the effect of various factors are small and therefore techniques for measuring the SAW velocity must be precise. The velocity of the surface acoustic waves is determined by measuring the distance between the SAW emitting and receiving areas and also by the respective propagation time of the acoustic wave. To measure the velocity one uses the SAW transmission and reception by utilizing contact piezoelectric transducers (Murav'Ev et al., 1996; ZHi et al., 2000; John- 
SON, 1993) or non-contact laser techniques (WAGNER, 1990). To determine the spatial distribution of the SAW velocity several measuring schemes are used. One of them consists in displacement of one of the transducers with respect to the other, measuring change of propagation time and distance. For a precise velocity measuring it is necessary to measure accurately the distance and the propagation time respectively. Since it is rather complicated to measure the distance precisely, another technique is used in which the emitting and receiving part of transducer are rigidly connected. Such a rigidly connected transducer is presented in Fig. 1, where SAWs are excited and recorded by the wedge transducers (JoHNsON, 1993).

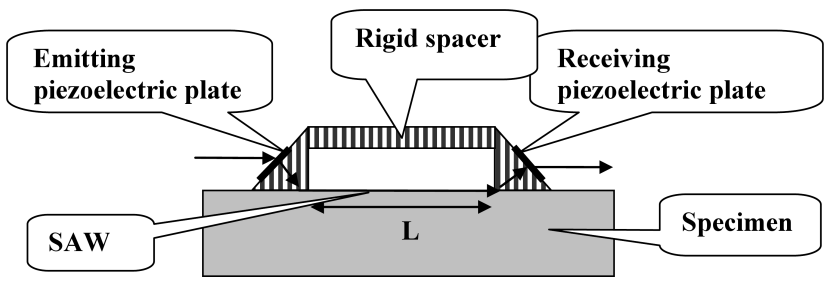

Fig. 1. Construction transducer with rigid spacer, $L$ is the measurement base.

In this case the distance between the emitting and receiving wedges remains constant and thus there is no need of its measuring. For measuring fulfillment it is enough to calibrate such a transducer by using specimens with a known velocity of SAW. A distance between the emitting and receiving wedges is the measurement base that determines the spatial resolution of the transducer.

By moving the transducer over the specimen surface it is possible to measure the spatial distribution of the SAW velocity. The average velocity of SAW at a distance equal to its base is determined by this transducer and the spatial resolution is defined by the value of the transducer base. In case when the value of spatial inhomogeneities of the specimen is less than the base of the transducer, they can be studied by displacing the transducer by a value less than the base dimensions. The value of this displacement is called the measurement step. The space resolution is improved and equal to measurement step. In this case the problem of the relation between the error of transducer displacement measurement and the error of the local SAW velocity determination is important. It is shown in this paper that the error of the transducer displacement measurement causes sufficiently small error of the local SAW velocity determination. It enables measurement of the transducer displacement with low precision. Therefore, preference of transducer with rigid spacer is remained because of the distance measurement simplicity. On the other hand this technique enables measurement step changes, which is important for the optimization of velocity measurement in inhomogeneous specimen.
The velocity spatial change is continuous, but measurements are carried out in discrete areas corresponding to the measurement step. The average velocity is measured in the area with a dimensions equal to the measurement step. The measurement step determines velocity spatial resolution and its measurement error. Therefore, the optimal measurement step must be chosen for concrete specimen. The method for measurement step size optimization is proposed. This method may be used for study the spatial inhomogeneous specimens. In this paper we consider the homogeneous specimen with the small inhomogeneous area. In this case the SAW velocity in inhomogeneous area is determined relatively to velocity in homogeneous area. Moreover, the measurement step is assumed constant for all specimen area. The proposed method is used for investigation of the inhomogeneous plastically deformed metal specimen.

\section{Analysis of the measuring technique}

Let us consider velocity measurement by using a transducer with rigid spacer. The propagation time of acoustic wave will be

$$
t=t_{p}+t_{s p}+t_{g}
$$

where $t_{p}$ is time of acoustic wave propagation through the transducer wedges, $t_{s p}$ is time of SAW propagation over the specimen surface, $t_{g}$ is time of acoustic wave propagation through the contact coupling layers between the transducer wedges and the specimens. With the help of calibration the values of $t_{p}, t_{g}$ and base $L$ for a concrete transducer are measured. After transducer calibration it is possible to find the SAW velocity in the studied specimen by measuring the propagation time $t$ according to the expression:

$$
V=\frac{L}{t_{s p}} .
$$

The value of $t_{s p}$ is obtained from expression (1) for $t_{p}$ and $t_{g}$ values known from calibration.

Let us consider the case when the transducer moves by a value less that its base. The time $t_{s p 0}$ of acoustic wave propagation over the specimen surface when the transducer is in position 0 and $t_{s p 1}-$ in position 1 , as shown in Fig. 2, will be:

$$
t_{s p 0}=t_{0}+t_{s}, \quad t_{s p 1}=t_{1}+t_{s},
$$

where $t_{0}$ is the time of acoustic pulse passing through the first area from which the transducer has been displaced in a null position, $t_{1}$ is propagation time in the second area to which the transducer has been displaced in other position, $t_{s}$ is time of the acoustic wave propagation through the specimen area, which is common 


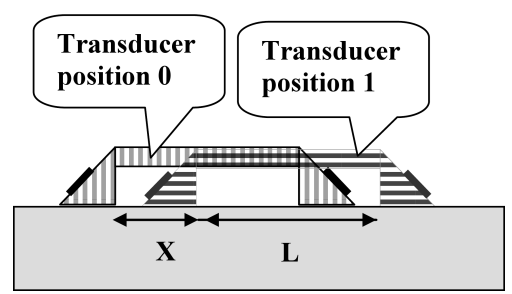

Fig. 2. Transducer in two positions, $X$ is measurement step.

for both locations of the transducer. Correspondingly we can write

$$
V_{0}=\frac{X}{t_{0}}, \quad V_{1}=\frac{X}{t_{1}},
$$

where $V_{0}, V_{1}$ are the SAW velocities in different areas from and to which the transducer has been displaced, $X$ is the value of the transducer displacement which is the measurement step.

If $t_{1}$ is represented as $t_{s p 1}-t_{s p 0}+\frac{X}{V_{0}}$, velocity $V_{1}$ can be expressed by using expression (4) as

$$
V_{1}=\frac{V_{0}}{\frac{t_{s p 1}-t_{s p 0}}{X} V_{0}+1} .
$$

Expression (5) allows us to determine the SAW velocity in one area of the specimen with relation to the other. Let us consider the error of velocity determination caused by the inaccurate evaluation of the measurement step $X$, since the necessity to measure it with a high accuracy can seriously complicate the measurement making the technique non-effective. Standard deviation of velocity $V_{1}$ evaluation caused by the error of the displacement value $X$ measurement will be:

$$
\frac{\delta V_{1}}{V_{1}}=\frac{1}{V_{1}} \sqrt{\left(\frac{\partial V_{1}}{\partial X}\right)^{2}} \delta X^{2}
$$

where $\delta X$ is the error transducer displacement determination. Proceeding from (5) and (6) it is possible to obtain:

$$
\frac{\delta V_{1}}{V_{1}}=\frac{1}{V_{1}} \frac{V_{0}^{2}}{\left(\frac{t_{s p 1}-t_{s p 0}}{X} V_{0}+1\right)^{2}} \frac{t_{s p 1}-t_{s p 0}}{X^{2}} \delta X
$$

and correspondingly:

$$
\frac{\delta V_{1}}{V_{1}}=\frac{V_{1}}{V_{1}} \frac{t_{s p 1}-t_{s p 0}}{t_{1}} \frac{\delta X}{X},
$$

taking into consideration that according to $(3) t_{s p 1}-$ $t_{s p 0}=t_{1}-t_{0}$, Eq. (8) can be written as:

$$
\frac{\delta V_{1}}{V_{1}}=\left(1-\frac{t_{0}}{t_{1}}\right) \frac{\delta X}{X} .
$$

As can be seen from this equation, the error of the velocity evaluation is determined by both the error of displacement $X$ determination and the value $1-\frac{t_{0}}{t_{1}}$. In practice in most cases the SAW velocity changes under different factors in only several percent. That means that $1-\frac{t_{0}}{t_{1}} \ll 1$ and the error of $X$ value determination causes a significantly less error of velocity $V$ determination. This can be explained by the fact that the velocity changes are measured only on one area of the specimen with relation to the other. Just this change is determined with the accuracy set by the accuracy of displacement value $X$ evaluation. When this value is insignificant the total error is also substantially less according to Eq. (9). This conclusion allows us to measure the transducer displacement with low accuracy. This fact simplifies greatly the measurement since the high-accuracy measuring can be fulfilled by using rather simple distance measuring tools. This is the advantage comparing to the technique where an emitting wedge is moved with respect to a receiving one.

The proposed technique allows one to determine the SAW velocity in one area of the specimen with respect to the other. This enables determining the spatial distribution of the SAW velocity in an inhomogeneous specimen.

Let us consider the use of this technique for specimen with spatially homogeneous properties, with a local-inhomogeneous area (see Fig. 3). The size of this inhomogeneity is less than the size of the transducer measurement base. For example, such inhomogeneities can appear as a result of local plastic deformation, welding, etc. The measuring technique consists in measuring fulfillment in different areas of the specimen under movement of the transducer from the homogeneous to inhomogeneous areas. This allows us to find the spatial distribution of the SAW velocity in the inhomogeneous region by using expression (5). As $V_{0}$ a known velocity of the specimen homogeneous region is used while $V_{1}$ corresponds to the average velocity of the specimen inhomogeneous part in the area equal by width to the displacement value $X$.

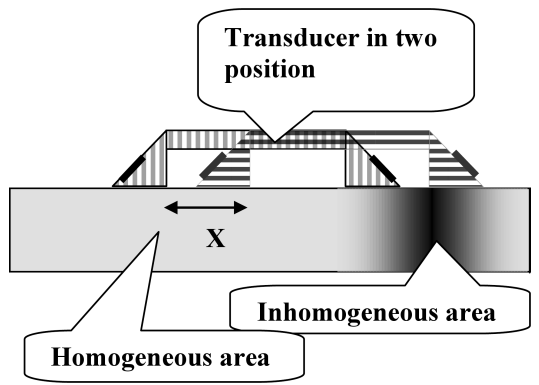

Fig. 3. Scheme measurement of the spatial distribution of the SAW velocity in inhomogeneous specimen.

\section{Optimization of a measuring step}

Another advantage of the technique is the possibility to change a measurement step during study. This 
advantage is very important during studing the inhomogeneous specimens. A measurement step determines a spatial resolution of the technique. Besides, a measurement step sets an error of local velocity measurement. For example, as seen from Eq. (9), an error of SAW velocity measurement, which is connected with inaccurate determination of the displacement value $X$, increases with its decrease. However, for insignificant spatial velocity changes this error is not dominant. The main sources of the error, mentioned in (MURAV'EV et al., 1996) are the acoustic instability of contact between transducer and the specimen and temperature instability of the measurement conditions. Taking into consideration that the error, related to the temperature instability, can be decreased due to thermostabilization and also due to other techniques, the main error still remains the error related to the instability of the acoustic contact. Thus, in the following considerations the main cause of the error is assumed to be the error related to the instability of acoustic contact. In the transducer used in our researches the instability of acoustic contact gives the time measurement error of $\delta t \sim 1$ ns. Based on Eq. (5), the error of the acoustic contact instability causes the velocity measurement error which is equal to:

$$
\delta V_{1}=\frac{2 V_{1}^{2}}{X} \delta t
$$

Since the time measurement error, related to the acoustic contact instability $\delta \mathrm{t}$, is constant, the relative error of velocity measurement will increase with the decrease of $X$ value. Thus, on the one hand, the value of the measurement step must be the minimum, proceeding from the high spatial resolution, and on the other hand it must be large, proceeding from the requirement of precise measurement. Therefore, an optimal value of a measurement step must be chosen. Evidently, a measurement step must be chosen on the basis of the characteristics of the studied specimens. An optimal step selection criterion should correlate its value with the measurement error value and as well as the properties that characterize a spatial inhomogeneity of the studied specimen. Proceeding from such considerations it is possible to propose the following criterion for the measurement step choice. As an optimal measurement step we can assume a minimum step for which the value of the velocity in the neighbor areas differs by a value larger that the measuring error. In this case the minimum accuracy, necessary for the inhomogeneities detection in the specimen under maximum spatial resolution, will be reached. Proceeding from this criterion for spatially homogeneous specimens where velocity is unchanged, a maximum large step must be chosen. In case of the specimens with large velocity gradient, the optimal measurement step will be small. To choose the optimal measurement step it is necessary to measure preliminary the SAW velocity spatial distribution in the specimen. Thus, the optimization of the measurement step can be carried out by consecutive measurements with different steps. The dependence graph of velocity spatial distribution with optimal step is chosen from these results. This graph is chosen using the optimization criterion. The plastically deformed steel specimens were studied by this technique.

\section{Research of plastically deformed specimens}

This technique was used for determining spatial distribution of SAW velocity in plastically deformed specimens of carbon steel $(\mathrm{C}=0.11-0.22 \%$, $\mathrm{Si}=0.15-0.3 \%, \mathrm{Mn}=0.4-0.65 \%, \mathrm{Ni}<0.3 \%, \mathrm{~S}<0.05 \%$, $\mathrm{P}<0.04 \%, \mathrm{Cr}<0.3 \%, \mathrm{~N}<0.008 \%, \mathrm{Cu}<0.3 \%, \mathrm{As}<0.08 \%$, $\mathrm{Fe} \sim 97 \%$ ). These steel specimens of length $220 \mathrm{~mm}$ and cross-section $10 \times 40 \mathrm{~mm}$ of the working part were subjected to tension up to fracture. After fracture the specimens were grinded and spatial distribution of the surface Rayleigh wave velocity was measured. The studied specimen is shown in Fig. 4. The left part of the specimen is fixed in a grip and this area is not deformed. The right part of the specimen with width $40 \mathrm{~mm}$ was subjected to tension up to fracture and in this case plastic deformation appeared in it.

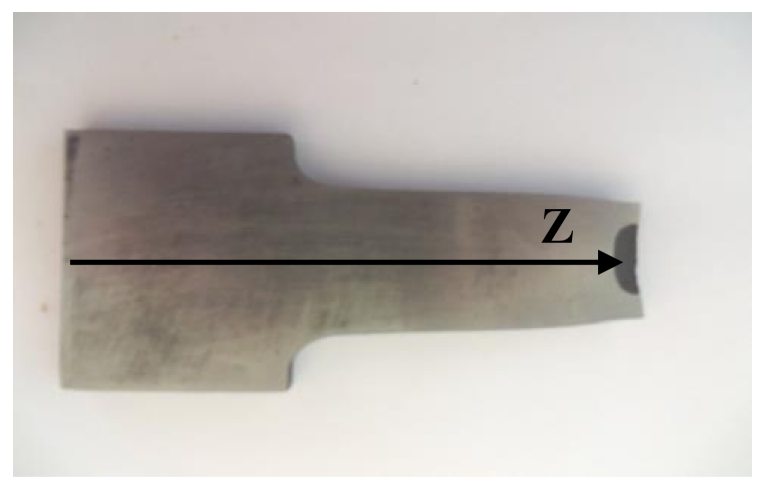

Fig. 4. Plastically deformed specimen.

Frequency of acoustic wave was $3 \mathrm{MHz}$. The driving signal of transmitting transducer was sine tone burst. This signal was produced by amplitude modulation of the signal from the sine generator with frequency $3 \mathrm{MHz}$. The width of the input driving signal was $2 \mu \mathrm{s}$. A transducer with a base of $70 \mathrm{~mm}$ was used. The transducer wedges were produced from the plexiglass. The wedges were coupled with the studied specimens by glycerine. The transducer was moved and its position was measured by the caliper. The transducer position error was $0.02 \mathrm{~mm}$. Signal from transducer was recorded by an oscillograph Tektronix TDS 1012. The received signal was digitized and recorded to the computer memory. Signals were registered for different positions of the transducer on the specimen and the correlation technique was utilized to determine the propagation time between them (Arattano, Marchi, 2005). 
According to the described technique the spatial distribution of the SAW velocity was determined. The transducer moved along the specimen surface from the non-deformed region to the deformed one. Acoustic wave propagated in the direction of tension that coincided with the direction of $Z$ axis. Measurement was fulfilled in the central part of the specimen. The obtained results are shown in Fig. 5. The measurement was carried out at three different measurement steps: 5,7 and $9 \mathrm{~mm}$. It is obvious that in the region close to the fracture area the velocity of SAW decreases. Since the main contribution to the error is done by an error related to acoustic contact instability the influence of other errors could be neglected.
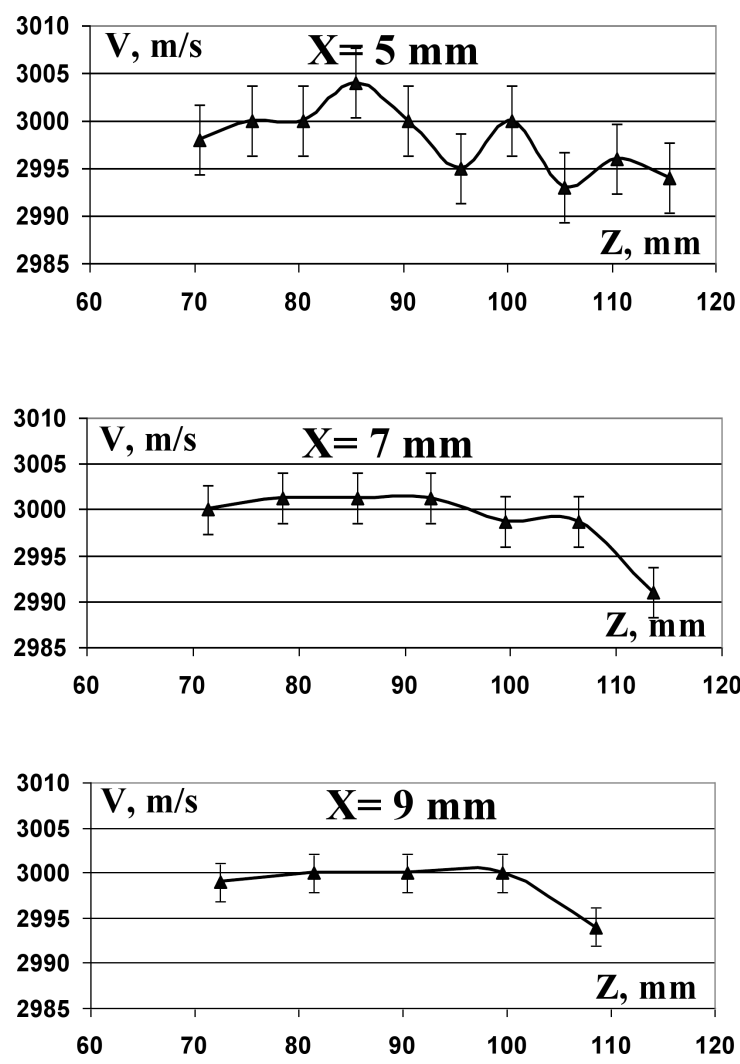

Fig. 5. Spatial distribution of the SAW velocity in plastically deformed sample with different measurement step.

According to the formulated criterion the optimal is a step at which a difference in the velocity between the neighbour regions exceeds the measuring error. The value of error of the SAW velocity calculated by Eq. (10) is shown in diagram (Fig. 5). This value depends on the value of the measurement step. As seen from the presented diagrams the optimal step of the measurement is different for the right and the left parts of the specimen. In the left part the optimal step is not reached. Since this is an area in which the velocity changes insignificantly with the change of the transducer position, the maximum measurement step will be optimal. An error in this case will be the minimum. In the right part of the specimen where velocity decreases the optimal measurement step is $X=7 \mathrm{~mm}$. Just at this value of the measurement step, the difference in the velocity for the neighbour areas of the specimen, which is larger than the measurement error, is observed. The measurement step of $5 \mathrm{~mm}$ is not optimal, because difference in the velocity for the neighbour areas of the specimen is less than the measurement error. The precision of measurement in this case is not satisfactory. On the other hand the measurement step of $9 \mathrm{~mm}$ provides spatial resolution lower than $7 \mathrm{~mm}$ and is not optimal too. Thus, the value $X=7 \mathrm{~mm}$ is optimal though it can be specified more precisely if necessary.

This example demonstrates the importance of the choice of optimal measurement step for determining spatial distribution of SAW velocity.

\section{Conclusions}

The technique for determination of spatial distribution of the SAW velocity by the contact transducer with rigid spacer is proposed. It is shown that the given technique does not require the measurement of the transducer position with a high accuracy and enables the measurement step change. The measurement step determines the measurement error of SAW velocity and the measurement spatial resolution. The measurement spatial resolution and precision are inversely proportional. The value of these parameters can be assessed by Eq. (10). The minimum measurement error is achieved for the maximum measurement step, which is limited by the size of the specimen homogeneous area or size of the transducer base. The minimum value of the spatial resolution is limited by the precision of measurement. The optimal values of these parameters should be determined for concrete specimen. The criterion for the measurement step optimization based on the measurement error estimation and specimen properties is proposed. Optimization of a measurement step during experimental study of the inhomogeneous plastically deformed specimen was carried out by this method.

\section{References}

1. Arattano M., Marchi L. (2005), Measurements of debris flow velocity through cross-correlation of instrumentation data, Natural Hazards and Earth System Sciences, 5, 137-142.

2. Hung-YAng Y., Jung-Ho C. (2003), NDE of metal damage: ultrasonics with a damage mechanics model, International Journal of Solids and Structures, 40, 72857298. 
3. Johnson C., Thompson R.B. (1993), The spatial resolution of Raileigh wave, acoustoelastic measurement of stress, Review of Progress in Quantutative Nondestraction Evalution, 12, 2121-2128, D.O. Thompson, D.E. Chimenti [Eds.], Plenum Press, New York.

4. LeWANDOWSKI J. (2001), Evalution of material parameters, texture and stress of prestressed polycrystalline aggregate from ultrasonic measurements, Archives of Acoustics, 26, 4, 305-329.

5. Li W., Coulson J., Aveson J.W., Smith R.J., Clark M., Somekh M.G., Sharples S.D. (2013), Orientation Characterisation of Aerospace Materials by Spatially Re- solved Acoustic Spectroscopy, 5th International Symposium on NDT in Aerospace, Singapore.

6. Murav'ev V.V., Zuev L.B., Komarov K.L. (1996), Velocity of Sound and Structure of Steel and Alloys [in Russian], Novosibirsk.

7. Wagner J.W. (1990), Optical detection of ultrasound. Physical Acoustics: Ultrasonic Measurement Methods, R.N. Thurston [Ed.], Boston, San Diego, New York, London, Sydney, Tokyo, Toronto, 19, 201-265.

8. Zhi W., Xiaojun Z., Yaodong C. (2000), Acoustoelastic determination of local surface stresses in polymethylmethacrylate, Applied Acoustics, 61, 477-485. 